HEALTH AND SAFETY EXECUTIVE (1989) Violence to Staff(IND (G) 1\89 M100). London: HMSO

ROYAL COLLEGE OF PSYCHIATRISTS (1998)

Management of Imminent Violence: Clinical Practice Guidelines for Support Mental Health Services. Occasional Paper OP41. London: Royal College of Psychiatrists.

David M. B. Christmas Senior House Officer in Psychiatry, Brian A, Kidd Consultant Psychiatrist, Forth Valley Primary Care NHS Trust, Cameron Stark Consultant in Public Health Highland Health Board

Sir: Davies (Psychiatric Bulletin, March 2001, 25, 89-91) suggests that further training is needed for psychiatric trainees in order to avoid being assaulted by patients and that further work is needed to distinguish the behaviour of those more experienced trainees who do not get assaulted. I heartily agree.

The police force cadets all get routine training in how to deal with violent and aggressive members of the public yet it is unusual to hear of this in psychiatric training. I suggest that yearly short sessions of 'break-away' and/or 'selfdefence' techniques with experienced trainers are introduced in trainees' timetables. From my own experience I have been lucky not to be assaulted, but I have had a few instances of patients trying to 'throw punches'. Even though my psychiatric experience has, I hope, taught me to detect when someone is about to 'boil over', I have to say the instance when the attack almost hit home was when I could not have predicted the event. Patients who hit out are usually not trained in any of the fighting arts and I feel only a little training is needed to escape being hit.

Stefan Kolowski Specialist Registrar in Old Age and Adult Psychiatry, Taunton and Somerset PartnershipTrust, Ryden House

\section{Urinary detection of olanzapine and its limitations - revisited}

Sir: Coates (Psychiatric Bulletin, May 2001, $\mathbf{2 5}, \mathbf{1 9 5}$ ) is correct in his assertion that a positive result (that is, the detection of olanzapine in urine) is open to various interpretations. This is the very reason why the value of this means of assessment of compliance is so questionable in clinical practice at present and is unlikely to prove better in the future, particularly for low doses of oral medication.

The quantitative assessment of urinary olanzapine is limited, as follows: $7 \%$ of the dose of olanzapine is excreted unchanged in urine. As urine concentrations vary with the volume of fluid intake, which can vary greatly in any individual from day-to-day, exact assessment of urine concentration would necessitate specific timed and total urine collection. Compliance with such a protocol is a most unlikely scenario, particularly in the very patients whose nonadherence is suspected. Even using creatinine concentrations for normalisa tion is inexact, and must require a number of assumptions to be made.

Coates' other scenario of a negative result, that is one that detects no olanzapine in the urine, is also debatable; even if no olanzapine could be detected in the urine one might speculate that no olanzapine had been taken recently, however even this is questionable given issues of assay sensitivity, drug stability in urine and fluid volume intake.

Taken together, none of the above results will deliver the level of precision of measurement that may be required for such purposes as mental health review tribunals as Coates has previously suggested (Psychiatric Bulletin, 2000, 24, 316).

On a more general point, awareness of a patient's adherence to medication is a major issue in the treatment of psychiatric patients, particularly if Mental Health Act legislation is to change to allow the community treatment of patients. Knowledge of a patient's true adherence is extremely difficult particularly in clinical practice.

Until technological developments improve for the quantitative assessment of drugs in body fluids other more direct observations of medication intake may be the best substitute for assessing adherence to medication. For example, one demonstrably successful method of achieving compliance and levels of compliance with methadone prescribing has been to directly observe patients taking their medication in liquid form. A similar trial is planned that involves community pharmacists observing community patients taking antipsychotic medications when they visit the pharmacy on a daily basis in return for a small daily reward.

Andrew Sandor Lecturer in Social and Community Psychiatry, Royal Free and University College Medical School

\section{Medical representation at mental health review tribunal}

Sir: Attendance at the mental health review tribunals (MHRTs) is the responsibility of the responsible medical officer (RMO) or the community RMO. In unforeseen circumstances where the RMO cannot attend, the tribunal expects him or her to arrange for the attendance of a medical colleague who is concerned with the patient's treatment, or sufficiently acquainted with the case to be able to report knowledgeably about the patient.

On occasions, unfortunately it is a new registrar on his first rotation in psychiatry who attends the tribunal in place of the RMO. Nervous, struggling with the use of English, the junior doctor makes it known to the tribunal that he or she has never been in this situation before. He or she may have interviewed the patient only a few times. He or she did not admit the patient and cannot explain why a patient with a history of four previous psychiatric admissions was admitted this time on Section 2. A more knowledgeable social worker helps him or her out with questions about the Mental Health Act. I wonder how many RMOs would have wished that on themselves at that stage of their psychiatric formation. The principle of 'do unto others as you would like to be done unto' seems to have been forgotten.

On other occasions it is a staff grade doctor who comes to represent his or her consultant in a Section 2 hearing. He or she has been called upon at such short notice that he or she attends with no written report. He or she reads the outpatient report or last letter to the general practitioner until the Chairman calls him or her to address the main issue: "Has your patient a mental disorder, the nature and degree of which warrants his or her detention in hospital for his or her own health and safety and for the safety of others?"

The above situations expose the College training to some criticisms by some of the tribunal members. This is an additional reason why the College should do something about it. In my view, the following measures ought to be taken to avoid such embarrassing situations:

(a) No junior doctor on his or her first rotation in psychiatry should be allowed to represent the $\mathrm{RMO}$ in a MHRT. Indeed only those with Part 1 of the Membership or a degree in psychiatry should be trained to do so.

(b) Consultants should take their junior doctors as observers to the MHRTs in order to expose them to the realities of the tribunal.

(c) Senior house officer/registrars prepare $54 \%$ of the reports (Ismail et al, 1998)، but these should be discussed and modified by the RMO before the report is sent to the tribunal office.

(d) There should always be a written medical report in Section 2 cases, no matter how brief the report. This is irrespective of who attends the tribunal, the RMO or his/her deputy.

Unless we keep our house in order these few cases will mar the excellent work done by a majority of RMOs. Junior doctors should be protected; the College owes it to them.

SMAIL, K., SMITH, S. \& MADEN, T. (1998) Mental health review tribunal medical reports. Psychiatric Bulletin, 22, 615-618.

B. N. Nwulu Consultant Psychiatrist and medical member of MHRT 